Pacific

Journal of

Mathematics

PERTURBATION OF DIFFERENTIAL OPERATORS ADMITTING A CONTINUOUS LINEAR RIGHT INVERSE ON ULTRADISTRIBUTIONS

RÜdiger W. Braun, Reinhold Meise, and B.A. Taylor 


\title{
PERTURBATION OF DIFFERENTIAL OPERATORS ADMITTING A CONTINUOUS LINEAR RIGHT INVERSE ON ULTRADISTRIBUTIONS
}

\author{
Rüdiger W. Braun, Reinhold Meise, and B.A. Taylor
}

Let $\boldsymbol{P}_{m}$ be a homogeneous polynomial of degree $\boldsymbol{m}$ in $n \geq 2$ variables for which the associated partial differential operator $P_{m}(D)$ admits a continuous linear right inverse on $C^{\infty}\left(\mathbb{R}^{n}\right)$. Examples suggest that then for each polynomial $Q$ of degree less than $m$ there exists a number $0<\beta<1$ such that the operator $\left(P_{m}+Q\right)(D)$ admits a continuous linear right inverse on the space of all $\omega_{\beta}$-ultradifferentiable functions on $\mathbb{R}^{n}$, where $\omega_{\beta}(t)=(1+t)^{\beta}$. The main result of the present paper is to determine the optimal value of $\beta$ for which the above holds for all perturbations $Q$ of a given degree in the case $n=3$. When $n>3$ sufficient conditions as well as necessary conditions of this type are presented, but there is a gap between them. The results are illustrated by several examples.

\section{Introduction.}

The problem of determining when a given partial differential operator $P(D)$ with constant coefficients admits a continuous linear right inverse on the space $\mathcal{E}(G)$ (respectively $\mathcal{D}^{\prime}(G)$ ) of all $C^{\infty}$ functions (respectively distributions) on an open set $G$ in $\mathbb{R}^{n}$ was solved in Meise, Taylor, and Vogt [11], where various equivalent characterizing conditions were given. In $[\mathbf{1 3}]$ these characterizations were extended to $\omega$-ultradifferentiable functions $\mathcal{E}_{(\omega)}(G)$ and to $\omega$-ultradistributions $\mathcal{D}_{(\omega)}^{\prime}(G)$ of Beurling type. Since all of these equivalent characterizations are rather involved, several attempts were made to derive other characterizations in terms of the symbol $P$ or its zero variety.

One way to attack this problem is based on a result from [15] which shows that if $P(D)$ admits a continuous linear right inverse on $\mathcal{E}_{(\omega)}\left(\mathbb{R}^{n}\right)$ then so does $P_{m}(D)$, where $P_{m}$ is the principal part of $P$. Thus, one might treat $P$ as a perturbation of its principal part $P_{m}$. In [4] this idea led to an explicit characterization of the homogeneous polynomials $P_{m}$ of degree $m$ in $n$ variables for which $\left(P_{m}+Q\right)(D)$ admits a continuous linear right inverse on $\mathcal{E}\left(\mathbb{R}^{n}\right)$ (or on $\mathcal{D}^{\prime}\left(\mathbb{R}^{n}\right)$ ) for each polynomial $Q$ of degree at most $m-1$. The main part of this characterization is that - up to a complex multiple 
- $P_{m}$ is a real polynomial of principal type; i.e., $P_{m}$ has real coefficients and $\operatorname{grad} P_{m}(x) \neq 0$ for $0 \neq x \in \mathbb{R}^{n}$.

Our aim in this paper is to refine the perturbation result just cited. The goal is to explain in terms of $P_{m}$ and $l=\operatorname{deg}(Q)<m$ the optimal choice of $\beta=\beta\left(l, P_{m}\right)$ so that $\left(P_{m}+Q\right)(D)$ has a continuous linear right inverse on $\mathcal{E}_{\left(\omega_{\beta}\right)}\left(\mathbb{R}^{n}\right)$ for all polynomials $Q$ of degree at most $l$. In dimension three, this is achieved in the following theorem:

Theorem 1.1. Let $P_{m} \in \mathbb{C}[x, y, z]$ be homogeneous of degree $m \geq 2$ and let $\nu=\max \left\{\operatorname{deg}\left(P_{m}\right)_{\theta}: \theta \in V\left(P_{m}\right) \cap S^{2}\right\}$, where $\left(P_{m}\right)_{\theta}$ denotes the localization of $P_{m}$ at $\theta$ (see Definition 3.4). For $0 \leq l<m$, let $\beta(l):=\max \left\{0,1-\frac{m-l}{\nu}\right\}$ and let $\mathcal{E}_{\left(\omega_{0}\right)}\left(\mathbb{R}^{3}\right):=\mathcal{E}\left(\mathbb{R}^{3}\right)$. Then $P_{m}(D)$ admits a continuous linear right inverse on $\mathcal{E}\left(\mathbb{R}^{3}\right)$ if and only if for each polynomial $Q \in \mathbb{C}[x, y, z], \operatorname{deg}(Q) \leq$ $l$, the operator $\left(P_{m}+Q\right)(D)$ admits a continuous linear right inverse on $\mathcal{E}_{\left(\omega_{\beta(l)}\right)}\left(\mathbb{R}^{3}\right)$.

Moreover, the number $\beta(l)$ is optimal in the following sense: If for some number $0 \leq \gamma<1$ the operator $\left(P_{m}+Q\right)(D)$ admits a continuous linear right inverse on $\mathcal{E}_{\left(\omega_{\gamma}\right)}\left(\mathbb{R}^{3}\right)$ for each $Q \in \mathbb{C}[x, y, z]$ with $\operatorname{deg} Q \leq l$, then $\gamma \geq \beta(l)$.

The proof of the theorem is carried out by establishing three new results about the Phragmén-Lindelöf condition $\operatorname{PL}\left(\mathbb{R}^{n}, \omega\right)$ (see Definition 2.4) that was shown in $[\mathbf{1 3}]$ to characterize the existence of a continuous linear right inverse for $P(D)$. First, we show (Proposition 3.3) that the condition can be localized to cones about the real points in $V\left(P_{m}\right) \cap S^{n-1}$. Second, we use the lemma of Boutroux-Cartan and Rouche's theorem to derive a sufficient condition for such Phragmén-Lindelöf conditions to hold. From this, we then derive a sufficient condition which ensures that for a given homogeneous polynomial $P_{m}$ in $n$ variables and for all perturbations $Q$ with $\operatorname{deg}(Q) \leq$ $l<m$, the variety $V\left(P_{m}+Q\right)$ satisfies $\operatorname{PL}\left(\mathbb{R}^{n}, \omega_{\beta}\right)$ where $\beta$ is given by the formula in Theorem 1.1. Third, we use a result from [4] to show that $\gamma \geq \beta(l)$ when $\left(P_{m}+Q\right)(D)$ admits a continuous linear right inverse on $\mathcal{E}_{\left(\omega_{\gamma}\right)}\left(\mathbb{R}^{n}\right)$ for each polynomial $Q$ of degree $l$, where $m-\nu \leq l<m$. The argument is based on the fact that the maximal degree of the localization of a homogeneous polynomial $P_{m} \in \mathbb{C}\left[z_{1}, \ldots, z_{n}\right]$ at the points in $V\left(P_{m}\right) \cap S^{n-1}$ greatly influences the existence of a continuous linear right inverse. The combination of these results then implies Theorem 1.1. We remark that the case $n=2$ is much simpler and was already known.

Our results also imply that we can extend the perturbation theorem from [4] to ultradifferentiable functions. Further, we show that under additional hypotheses on the localization at a singular point $\xi \in V\left(P_{m}\right) \cap S^{2}$, a better result concerning Phragmén-Lindelöf conditions in cones can be obtained (Lemma 4.1). Finally, quite a number of examples are provided. They also show the known effect that for a fixed polynomial $Q$, the operator $\left(P_{m}+Q\right)(D)$ may do better than predicted by Theorem 1.1. Namely, it 
may have a continuous linear right inverse on $\mathcal{E}_{\left(\omega_{\sigma}\right)}\left(\mathbb{R}^{n}\right)$ for some $\sigma<\beta$. When $n=3$, the optimal value for $\sigma$ can be determined. However, this is a much more complicated procedure, based on hyperbolicity considerations, for which we refer to our forthcoming paper [6].

\section{Preliminaries.}

In this preliminary section we introduce the basic definitions, notation, and a few results which will be used subsequently.

Throughout this paper, |.| denotes the Euclidean norm on $\mathbb{C}^{n}$ and $B^{n}(\xi, r)$ denotes the ball of center $\xi$ and radius $r$ in $\mathbb{C}^{n}$.

Definition 2.1. Let $\omega:] 0, \infty[\rightarrow] 0, \infty[$ be continuous and increasing and assume that it has the following properties:

$(\alpha) \omega(2 t)=O(\omega(t))$,

( $\beta) \int_{1}^{\infty} \frac{\omega(t)}{t^{2}} d t<\infty$,

$(\gamma) \log t=O(\omega(t))$ as $t$ tends to infinity,

$(\delta) x \mapsto \omega\left(e^{x}\right)$ is convex.

Then its radial extension to $\mathbb{C}^{n}$, defined by $\omega: z \mapsto \omega(|z|), z \in \mathbb{C}^{n}$, will be called a weight function. Throughout this paper we assume that $\omega(0) \geq 1$. It is easy to check that this can be assumed without loss of generality.

Example 2.2. Examples of weight functions are

(a) $\omega_{0}(t)=\log (e+t)$,

(b) $\omega_{\alpha}(t)=(1+t)^{\alpha}$ for $0<\alpha<1$.

Definition 2.3. Let $V$ be an algebraic variety in $\mathbb{C}^{n}$ and $\Omega$ an open subset of $V$. A function $u: \Omega \rightarrow[-\infty, \infty$ [ will be called plurisubharmonic if it is locally bounded above, plurisubharmonic in the usual sense on $\Omega_{\text {reg }}$, the set of all regular points of $V$ in $\Omega$, and satisfies

$$
u(z)=\limsup _{\xi \in \Omega_{\mathrm{reg}}, \xi \rightarrow z} u(\xi)
$$

at the singular points of $V$ in $\Omega$. $\operatorname{By} \operatorname{PSH}(\Omega)$ we denote the set of all plurisubharmonic functions on $\Omega$.

Definition 2.4. Let $V \subset \mathbb{C}^{n}$ be an algebraic variety and let $\omega$ be a weight function. Then $V$ satisfies the condition $\operatorname{PL}\left(\mathbb{R}^{n}, \omega\right)$ if the following holds:

There exists $A \geq 1$ such that for each $\rho>1$ there exists $B>0$ such that each $u \in \operatorname{PSH}(V)$ satisfying $(\alpha)$ and $(\beta)$ also satisfies $(\gamma)$, where:

( $\alpha) u(z) \leq|\operatorname{Im} z|+O(\omega(z)), z \in V$

(ß) $u(z) \leq \rho|\operatorname{Im} z|, z \in V$,

$(\gamma) u(z) \leq A|\operatorname{Im} z|+B \omega(z), z \in V$.

Phragmén-Lindelöf conditions and continuous linear right inverses 2.5. To explain the significance of the condition $\operatorname{PL}\left(\mathbb{R}^{n}, \omega\right)$, let 
$n \geq 2$, let $P(z)=\sum_{|\alpha| \leq m} a_{\alpha} z^{\alpha}$ be a complex polynomial of degree $m>0$, and let

$$
V(P):=\left\{z \in \mathbb{C}^{n}: P(-z)=0\right\}
$$

denote its zero variety. Then $V(P)$ satisfies $\operatorname{PL}\left(\mathbb{R}^{n}, \omega\right)$ if and only if the linear partial differential operator

$$
P(D): \mathcal{E}_{(\omega)}\left(\mathbb{R}^{n}\right) \rightarrow \mathcal{E}_{(\omega)}\left(\mathbb{R}^{n}\right), \quad P(D) f:=\sum_{|\alpha| \leq m} a_{\alpha} i^{-|\alpha|} \frac{\partial^{|\alpha|} f}{\partial x^{\alpha}}
$$

admits a continuous linear right inverse, where $\mathcal{E}_{(\omega)}\left(\mathbb{R}^{n}\right)$ is the Fréchet space of all $\omega$-ultradifferentiable functions of Beurling type (see [2]). This follows from the general characterization in Meise, Taylor, and Vogt [15]. Recall that $\mathcal{E}_{\left(\omega_{0}\right)}\left(\mathbb{R}^{n}\right)=C^{\infty}\left(\mathbb{R}^{n}\right)$ and that in this case the characterization of the existence of continuous linear right inverses was already obtained in Meise, Taylor, and Vogt [12]. Note also that Palamodov [17] proved that a differential complex of $C^{\infty}$-functions over $\mathbb{R}^{n}$ splits if and only if the associated varieties satisfy $\mathrm{PL}\left(\mathbb{R}^{n}, \omega_{0}\right)$.

From [12], Lemma 2.9, we recall the following lemma:

Lemma 2.6. For each $n \in \mathbb{N}$ the function $H: \mathbb{C}^{n} \rightarrow \mathbb{R}$, defined as $H(z):=$ $\frac{1}{2}\left((\operatorname{Im} z)^{2}-(\operatorname{Re} z)^{2}\right)$ is plurisubharmonic and has the following properties:

(a) $H(z) \leq|\operatorname{Im} z|, \quad|z| \leq 1$,

(b) $H(z) \leq|\operatorname{Im} z|-\frac{1}{2}, \quad|z|=1$,

(c) $H(x) \leq 0, \quad x \in \mathbb{R}^{n}$,

(d) $H(i y) \geq 0, \quad y \in \mathbb{R}^{n}$.

Definition 2.7. For $d=\left(d_{1}, \ldots, d_{n}\right) \neq(0, \ldots, 0)$ with $d_{j} \in \mathbb{N}_{0}, 1 \leq j \leq n$, a nonzero polynomial $P \in \mathbb{C}\left[z_{1}, \ldots, z_{n}\right]$ is said to be $d$-quasihomogeneous of $d$-degree $m \geq 0$ if

$$
P(z)=\sum_{\langle d, \alpha\rangle=m} a_{\alpha} z^{\alpha}, \quad z \in \mathbb{C}^{n},
$$

where $\langle d, \alpha\rangle=\sum_{j=1}^{n} d_{j} \alpha_{j}$. The zero polynomial is considered to be $d$ quasihomogeneous of $d$-degree $-\infty$.

Combining Lemma 3.2 and Lemma 3.6 from [4] we get the following lemma:

Lemma 2.8. For $n \geq 2$ let $P \in \mathbb{C}\left[z_{1}, \ldots, z_{n}\right]$ be d-quasihomogeneous of $d$-degree $m$ and let $Q \in \mathbb{C}\left[z_{1}, \ldots, z_{n}\right]$ be the sum of $d$-quasihomogeneous polynomials of d-degrees less than $m$. Assume that for some $k, 1 \leq k<n$, the following conditions are fulfilled:

(1) $d_{1}=\cdots=d_{k}<d_{j}$ for $j>k$,

(2) there exists $\zeta=\left(\zeta^{\prime}, \zeta^{\prime \prime}\right) \in \mathbb{C}^{k} \times \mathbb{R}^{n-k}$ satisfying $P(\zeta)=0$ and $\zeta^{\prime \prime} \neq 0$, 
(3) if $P\left(z^{\prime}, \zeta^{\prime \prime}\right)=0$ then $\operatorname{Im} z^{\prime} \neq 0$.

If $V(P+Q)$ satisfies $\mathrm{PL}\left(\mathbb{R}^{n}, \omega\right)$ for some weight function $\omega$ and $D:=$ $\max \left\{d_{j}: \zeta_{j} \neq 0\right\}$, then $\omega$ satisfies $t^{d_{1} / D}=O(\omega(t))$ as $t$ tends to infinity.

\section{Main results.}

The aim of this section is to derive conditions which imply that for a homogeneous poynomial $P \in \mathbb{C}\left[z_{1}, \ldots, z_{n}\right]$ for which $V(P)$ satisfies $\operatorname{PL}\left(\mathbb{R}^{n}, \omega_{0}\right)$ the variety $V(P+Q)$ satifies $\operatorname{PL}\left(\mathbb{R}^{n}, \omega_{\beta(l)}\right)$ for all polynomials $Q$ of degree $l<m$. The number $\beta(l)$ will be shown to be sharp.

Throughout this section we assume $n \geq 2$ unless other assumptions are made.

Instead of working with the property $\mathrm{PL}\left(\mathbb{R}^{n}, \omega\right)$ as it is given in Definition 2.4, it is often easier to consider the intersection of the variety $V$ with cones. This will be made more precise in Proposition 3.3. To formulate this proposition, recall that for a point $\xi$ in the unit sphere $S^{n-1} \subset \mathbb{R}^{n}$, a set $M$ with $\bar{M} \subset B^{n}(0,1)$, and $r>0$ the cone $\Gamma(\xi, M, r)$ around the ray generated by $\xi$ with profile $M$, truncated at $r$, is defined as

$$
\Gamma(\xi, M, r):=\bigcup_{t>r} t(\xi+M) .
$$

Definition 3.1. For $P \in \mathbb{C}\left[z_{1}, \ldots, z_{n}\right] \backslash \mathbb{C}$ let $V:=V(P)$, let $\omega$ be a weight function, and let $\Gamma:=\Gamma(\xi, G, r)$ be a cone for which $G$ is an open neighborhood of zero in $\mathbb{C}^{n}$. We say that $V$ satisfies the condition $\operatorname{PL}(V(P), \Gamma, \omega)$ if there exist a compact set $K \subset G$ which is a neighborhood of zero and numbers $A_{1} \geq 1$ and $r_{1} \geq r$ such that for each $\rho>0$ there exists $B_{\rho}$ such that for each $u \in \operatorname{PSH}(V \cap \Gamma)$ the following two conditions:

( $\alpha) u(z) \leq|z|, z \in V \cap \Gamma$,

( $\beta) u(z) \leq \rho|\operatorname{Im} z|, z \in V \cap \Gamma$,

imply

( $\gamma) u(z) \leq A_{1}|\operatorname{Im} z|+B_{\rho} \omega(z), z \in V \cap \Gamma\left(\xi, K, r_{1}\right)$.

Lemma 3.2. For a polynomial $P \in \mathbb{C}\left[z_{1}, \ldots, z_{n}\right] \backslash \mathbb{C}$ denote by $P_{m}$ its principal part and let $\omega$ be a weight function. If $V(P)$ satisfies the condition $\mathrm{PL}\left(\mathbb{R}^{n}, \omega\right)$ then for each $\xi \in V\left(P_{m}\right) \cap S^{n-1}, r \geq 1$, and each open zero neighborhood $G$ with $\bar{G} \subset B^{n}(0,1)$, the variety $V(P)$ has the property $\operatorname{PL}(V(P), \Gamma(\xi, G, r), \omega)$.

Proof. Fix $\xi \in V\left(P_{m}\right) \cap S^{n-1}, r \geq 1$, and $G$ as in the statement of the lemma. Then fix a compact zero neighborhood $K \subset G$, choose $0<\eta<1$ so small that $K+B(0,2 \eta) \subset G$ and note that $\max \{|z|: z \in K\} \leq 1$. Next fix $u \in \operatorname{PSH}(V(P) \cap \Gamma(\xi, G, r))$ and assume that $u$ satisfies Conditions $3.1(\alpha)$ and $(\beta)$. Now fix 


$$
z_{0}=t_{0} \xi+t_{0} w_{0} \in \Gamma(\xi, K, r)
$$

and distinguish the following two cases:

Case 1: $\left|\operatorname{Im} z_{0}\right| \geq \eta t_{0}$.

Then $\left|z_{0}\right| \leq t_{0}\left|\xi+w_{0}\right| \leq 2 t_{0}$, the present hypothesis, and Condition $3.1(\alpha)$ on $u$ imply

$$
u\left(z_{0}\right) \leq\left|z_{0}\right| \leq 2 t_{0} \leq \frac{2}{\eta}\left|\operatorname{Im} z_{0}\right|
$$

Case 2: $\left|\operatorname{Im} z_{0}\right|<\eta t_{0}$.

Then note that for each $z \in B\left(\operatorname{Re} z_{0}, \eta t_{0}\right)$ the present hypothesis and the choice of $\eta$ imply

$$
z-t_{0} \xi=z-\operatorname{Re} z_{0}-i \operatorname{Im} z_{0}+z_{0}-t_{0} \xi \in t_{0} B(0,2 \eta)+t_{0} K \subset t_{0} G .
$$

Hence we can define $\varphi: V(P) \rightarrow[-\infty, \infty[$ by

$$
\varphi(z):=\max \left\{\frac{\eta}{9} u(z)+\eta t_{0} H\left(\frac{z-\operatorname{Re} z_{0}}{\eta t_{0}}\right),|\operatorname{Im} z|\right\}, z \in V \cap B\left(\operatorname{Re} z_{0}, \eta t_{0}\right)
$$

and by $\varphi(z):=|\operatorname{Im} z|$ elsewhere on $V$, where $H$ denotes the function defined in Lemma 2.6. To see that $\varphi$ is plurisubharmonic on $V$, note that for $z \in V \cap \partial B\left(\operatorname{Re} z_{0}, \eta t_{0}\right)$ the above estimate for $z_{0}$ implies

$$
|z|=\left|z-\operatorname{Re} z_{0}+\operatorname{Re} z_{0}\right| \leq \eta t_{0}+\left|z_{0}\right| \leq 3 t_{0} .
$$

Hence Condition $3.1(\alpha)$ for $u$ gives $u(z) \leq 3 t_{0}$. By the properties of $H$, this implies

$$
\frac{\eta}{9} u(z)+\eta t_{0} H\left(\frac{z-\operatorname{Re} z_{0}}{\eta t_{0}}\right) \leq \frac{\eta}{3} t_{0}+|\operatorname{Im} z|-\frac{\eta}{2} t_{0}<|\operatorname{Im} z|
$$

for each $z \in V(P) \cap \partial B\left(\operatorname{Re} z_{0}, \eta t_{0}\right)$. Thus, $\varphi$ is plurisubharmonic on $V(P)$. From $3.1(\beta)$ and the properties of $H$ it follows that

$$
\varphi(z) \leq\left(\frac{\eta}{9} \rho+1\right)|\operatorname{Im} z|, \text { and } \varphi(z)=|\operatorname{Im} z|+O(1), \quad z \in V .
$$

Since $V(P)$ satisfies $\operatorname{PL}\left(\mathbb{R}^{n}, \omega\right)$, we conclude from these estimates the existence of $A \geq 1$ depending only on $V(P)$, and of $B$, depending on $\rho$, such that

$$
\varphi(z) \leq A|\operatorname{Im} z|+B \omega(z), \quad z \in V .
$$

Evaluating this estimate at $z_{0}$ and using the properties of $H$ together with the definition of $\varphi$, we get

$$
A\left|\operatorname{Im} z_{0}\right|+B \omega\left(z_{0}\right) \geq \varphi\left(z_{0}\right) \geq \frac{\eta}{9} u\left(z_{0}\right)+\eta t_{0} H\left(\frac{i \operatorname{Im} z_{0}}{\eta t_{0}}\right) \geq \frac{\eta}{9} u\left(z_{0}\right),
$$


and hence

$$
u\left(z_{0}\right) \leq \frac{9 A}{\eta}\left|\operatorname{Im} z_{0}\right|+\frac{9 B}{\eta} \omega\left(z_{0}\right) .
$$

Both cases together show that $u$ satisfies Condition $3.1(\gamma)$ with $A_{1}:=\frac{9 A}{\eta}$ and $B_{\rho}:=\frac{9 B}{\eta}$.

Proposition 3.3. Let $P \in \mathbb{C}\left[z_{1}, \ldots, z_{n}\right]$ be a polynomial of degree $m \geq 1$ and denote by $P_{m}$ its principal part. Then for a given weight function $\omega$ the following two conditions are equivalent:

(a) $V(P)$ satisfies $\mathrm{PL}\left(\mathbb{R}^{n}, \omega\right)$.

(b) $V\left(P_{m}\right)$ satisfies $\mathrm{PL}\left(\mathbb{R}^{n}, \omega_{0}\right)$ and for each $\xi \in V\left(P_{m}\right) \cap S^{n-1}$ there exist an open neighborhood $G_{\xi}$ of zero and $r_{\xi}>0$ such that $V(P)$ satisfies $\operatorname{PL}\left(V(P), \Gamma\left(\xi, G_{\xi}, r_{\xi}\right), \omega\right)$.

Proof. (a) $\Rightarrow(\mathrm{b})$ : If $V(P)$ satisfies $\mathrm{PL}\left(\mathbb{R}^{n}, \omega\right)$ then also $V\left(P_{m}\right)$ satisfies $\operatorname{PL}\left(\mathbb{R}^{n}, \omega_{0}\right)$ by Meise, Taylor, and Vogt $[\mathbf{1 5}]$, Theorem 4.1. Hence the first condition of (b) is fulfilled. The second one holds by Lemma 3.2.

(b) $\Rightarrow\left(\right.$ a): Since $V\left(P_{m}\right)$ satisfies $\operatorname{PL}\left(\mathbb{R}^{n}, \omega_{0}\right)$ by the present hypothesis, it follows from [15], Theorem 3.13, and Meise, Taylor, and Vogt [12], Theorem 5.1, that $V(P)$ satisfies Condition (RPL) defined in [12], 2.2. This means that there exists $A_{0} \geq 1$ such that for each $\rho>0$ there exists $B_{\rho}>0$ such that each $u \in \operatorname{PSH}(V(P))$ satisfying

$$
u(z) \leq|z|+o(|z|) \text { and } u(z) \leq \rho|\operatorname{Im} z|, z \in V(P)
$$

also satisfies

$$
u(z) \leq A_{0}|z|+B_{\rho}, z \in V(P) .
$$

From this we get in particular that each $u \in \operatorname{PSH}(V(P))$ which satisfies Conditions $2.4(\alpha)$ and $(\beta)$ of $\mathrm{PL}\left(\mathbb{R}^{n}, \omega\right)$ already satisfies (3.1). Consequently, $v(z):=\frac{1}{A_{0}} u(z)-B_{\rho}$ satisfies Conditions $3.1(\alpha)$ and $(\beta)$ with $\rho^{\prime}:=\frac{\rho}{A_{0}}$ in any cone $\Gamma\left(\xi, G_{\xi}, r_{\xi}\right), \xi \in V\left(P_{m}\right) \cap S^{n-1}$. Therefore we can use the hypothesis and a compactness argument to conclude similarly as in the proof of Meise and Taylor [10], Proposition 4.5, that there exist $A_{1} \geq 1$ and $C_{\rho}>0$ such that $u$ satisfies

$$
u(z) \leq A_{1}|\operatorname{Im} z|+C_{\rho} \omega(z), \quad z \in V(P) .
$$

Hence (a) holds.

To apply Proposition 3.3 we will use the following lemma, which is the key step for our positive results. To formulate it we need the following definitions: 
Definition 3.4. Let $P \in \mathbb{C}\left[z_{1}, \ldots, z_{n}\right]$ and $\theta \in \mathbb{C}^{n}$ be given. Then the localization $P_{\theta}$ of $P$ at $\theta$ is defined as the lowest order nonvanishing homogeneous polynomial in the Taylor series expansion of $P$ at $\theta$.

Definition 3.5. For $P \in \mathbb{C}\left[z_{1}, \ldots, z_{n}\right]$ the variety $V(P)$ is said to be locally hyperbolic at $\xi \in V(P) \cap \mathbb{R}^{n}$ if there exist a projection $\pi: \mathbb{C}^{n} \rightarrow \mathbb{C}^{n}$ and an open neighborhood $U$ of $\xi$ such that the following conditions are satisfied:

(a) $\operatorname{ker} \pi$ and $\operatorname{im} \pi$ are spanned by real vectors, $\operatorname{dim} \operatorname{ker} \pi=1$, and $(\operatorname{ker} \pi) \cap$ $V\left(P_{\xi}\right)=\{0\}$.

(b) Whenever $z \in V(P) \cap U$ and $\pi(z)$ is real then $z$ is real.

Lemma 3.6. Let $P_{m} \in \mathbb{C}\left[z_{1}, \ldots, z_{n}\right]$ be homogeneous of degree $m \geq 2$. Assume $P_{m}(\xi)=0, \operatorname{deg}\left(P_{m}\right)_{\xi}=\mu$, and $\left(P_{m}\right)_{\xi}(0, \ldots, 0,1) \neq 0$ for $\xi=$ $(1,0, \ldots, 0)$ and define $\beta(l):=\max \left(0,1-\frac{m-l}{\mu}\right)$ for $0 \leq l<m$. Then for each $Q \in \mathbb{C}\left[z_{1}, \ldots, z_{n}\right], \operatorname{deg} Q \leq l<m$, there exist $\eta, \sigma>0$ and $R, C>1$ such that the following holds:

(a) For each $\left(z_{1}, z^{\prime}\right) \in \mathbb{C}^{n-1}$ satisfying $\left|\frac{z_{1}}{\left|z_{1}\right|}-1\right|<\eta,\left|z_{1}\right|>R$, and $\left|z^{\prime}\right|<\eta\left|z_{1}\right|$, and for each $\zeta \in \mathbb{C}$ satisfying $|\zeta|<\sigma\left|z_{1}\right|$ and $\left(P_{m}+\right.$ $Q)\left(z_{1}, z^{\prime}, \zeta\right)=0$, there exists $w \in \mathbb{C}$ satisfying $|w|<\sigma\left|z_{1}\right|, P_{m}\left(z_{1}, z^{\prime}\right.$, $w)=0$, and

$$
|\zeta-w| \leq C\left|z_{1}\right|^{\beta(l)} .
$$

(b) If $V\left(P_{m}\right)$ is locally hyperbolic at $\xi$ with respect to the projection $\pi$ : $\left(z^{\prime \prime}, z_{n}\right) \mapsto\left(z^{\prime \prime}, 0\right)$, then the parameters $\eta, \sigma, R$ and $C$ in (a) can be chosen in such a way that for each $\left(z_{1}, z^{\prime}, \zeta\right)$ satisfying $\left(P_{m}+Q\right)\left(z_{1}, z^{\prime}, \zeta\right)=$ 0 and $\left|\frac{z_{1}}{\left|z_{1}\right|}-1\right|<\eta,\left|z_{1}\right|>R,\left|z^{\prime}\right|<\eta\left|z_{1}\right|,|\zeta|<\sigma\left|z_{1}\right|$, and $\left(z_{1}, z^{\prime}\right)$ real, we have

$$
|\operatorname{Im} \zeta| \leq C\left|z_{1}\right|^{\beta(l)} .
$$

Proof. The present hypotheses imply for the Taylor series expansion of $P_{m}$ at $\xi$ (see [3], Lemma 3.9)

$$
P_{m}\left(z_{1}, z^{\prime}, z_{n}\right)=\sum_{j=\mu}^{m} z_{1}^{m-j} p_{j}\left(z^{\prime}, z_{n}\right)
$$

where $p_{j}$ is either homogeneous of degree $j$ or identically zero, and where $\left(P_{m}\right)_{\xi}\left(z_{1}, z^{\prime}, z_{n}\right)=p_{\mu}\left(z^{\prime}, z_{n}\right)$. From this expansion and the hypotheses we get

$$
P_{m}\left(1,0^{\prime}, z_{n}\right)=\sum_{j=\mu}^{m} z_{n}^{j} p_{j}\left(0^{\prime}, 1\right)=z_{n}^{\mu}\left(\left(P_{m}\right)_{\xi}\left(0,0^{\prime}, 1\right)+\sum_{j=\mu+1}^{m} z_{n}^{j-\mu} p_{j}\left(0,0^{\prime}, 1\right)\right) .
$$

Hence we can choose $\sigma>0$ such that $z_{n} \mapsto P_{m}\left(1,0^{\prime}, z_{n}\right)$ has exactly $\mu$ zeros in the disk $B^{1}(0, \sigma)$ and does not vanish on $\partial B^{1}(0, \sigma)$. Hence it follows from 
the Weierstraß preparation theorem that we can choose $\eta>0$ such that for $\left(z_{1}, z^{\prime}, z_{n}\right) \in G:=B^{1}(1, \eta) \times B^{n-2}(0, \eta) \times B^{1}(0, \sigma)$ we have

$$
\begin{aligned}
P_{m}\left(z_{1}, z^{\prime}, z_{n}\right) & =U\left(z_{1}, z^{\prime}, z_{n}\right) \sum_{j=0}^{\mu} z_{n}^{j} c_{j}\left(z_{1}, z^{\prime}\right) \\
& =U\left(z_{1}, z^{\prime}, z_{n}\right) \prod_{j=1}^{\mu}\left(z_{n}-\beta_{j}\left(z_{1}, z^{\prime}\right)\right),
\end{aligned}
$$

where $U$ is a holomorphic function which does not vanish on $G$. In fact, shrinking $\eta$ if necessary, we may assume that there exists $\alpha>0$ such that $|U(z)|>\alpha$ for all $z \in G$. We also may assume $\left|\beta_{j}\left(z_{1}, z^{\prime}\right)\right| \leq \sigma / 2$ for $\left(z_{1}, z^{\prime}\right) \in$ $B^{1}(1, \eta) \times B^{n-2}(0, \eta)$ and $1 \leq j \leq \mu$. Next note that by the homogeneity of $P_{m}, U$ is also homogeneous and extends holomorphically to the cone

$$
\begin{aligned}
\Gamma:=\left\{\left(z_{1}, z^{\prime}, z_{n}\right) \in \mathbb{C}^{n}:\right. & \left|\frac{z_{1}}{\left|z_{1}\right|}-1\right|<\eta, \\
& \left.\left|z^{\prime}\right|<\eta\left|z_{1}\right|,\left|z_{n}\right|<\sigma\left|z_{1}\right|, \text { and }\left|z_{1}\right|>1\right\} .
\end{aligned}
$$

For $z=\left(z_{1}, z^{\prime}, z_{n}\right) \in \Gamma$ we have

$$
|U(z)|=\left|z_{1}\right|^{m-\mu}\left|U\left(\frac{z}{\left|z_{1}\right|}\right)\right| \geq \alpha\left|z_{1}\right|^{m-\mu} .
$$

Also by homogeneity the functions $\beta_{j}$ extend to the cone

$$
\Gamma^{\prime}:=\left\{\left(z_{1}, z^{\prime}\right) \in \mathbb{C}^{n-1}:\left|\frac{z_{1}}{\left|z_{1}\right|}-1\right|<\eta, \text { and }\left|z^{\prime}\right|<\eta\left|z_{1}\right|\right\} .
$$

For $\left(z_{1}, z^{\prime}\right) \in \Gamma^{\prime}$ define $F\left(z_{1}, z^{\prime}, z_{n}\right):=\prod_{j=1}^{\mu}\left(z_{n}-\beta_{j}\left(z_{1}, z^{\prime}\right)\right)$ and note that by the Lemma of Boutroux-Cartan (see Levin [9], Theorem I.10) the following holds: For each $\left(z_{1}, z^{\prime}\right) \in \Gamma^{\prime}$ and each $\delta>0$ there exist finitely many disks $D_{l}\left(z_{1}, z^{\prime}\right), 1 \leq l \leq d\left(z_{1}, z^{\prime}\right)$, for which the sum of the radii is at most $2 \delta$, such that

$$
\left|F\left(z_{1}, z^{\prime}, z_{n}\right)\right| \geq\left(\frac{\delta}{e}\right)^{\mu} \quad \text { whenever } z_{n} \in \mathbb{C} \backslash \bigcup_{l=1}^{d} D_{l} .
$$

We may assume that the $D_{l}\left(z_{1}, z^{\prime}\right)$ are constructed as in the proof that is given in [9]; then each $D_{l}\left(z_{1}, z^{\prime}\right)$ contains at least one zero of $F\left(z_{1}, z^{\prime}, \cdot\right)$. Now fix $Q \in \mathbb{C}\left[z_{1}, \ldots, z_{n}\right], \operatorname{deg} Q \leq l<m$. Then it is easy to check that there exists a constant $M>1$ such that

$$
|Q(z)| \leq M\left|z_{1}\right|^{l}, \quad z \in \Gamma .
$$


Choose $r>1$ and $R>1$ so large that $\alpha r^{\mu}>M$ and such that $4 e r t^{\beta(l)}<\frac{\sigma}{2} t$ for $t \geq R$. Next fix $\left(z_{1}, z^{\prime}\right) \in \Gamma^{\prime}$ satisfying $\left|z_{1}\right|>R$ and let

$$
\delta:=e r\left|z_{1}\right|^{\beta(l)} \text {. }
$$

Then it follows from (3.2), (3.3), (3.4), and our choice of $r$ that for each $z_{n} \in \mathbb{C} \backslash \bigcup_{l=1}^{d} D_{l}\left(z_{1}, z^{\prime}\right)$ satisfying $\left|z_{n}\right|<\sigma\left|z_{1}\right|$ we have

$$
\begin{aligned}
\left|P_{m}\left(z_{1}, z^{\prime}, z_{n}\right)\right| & \geq \alpha\left|z_{1}\right|^{m-\mu}\left(\frac{\delta}{e}\right)^{\mu} \\
& =\alpha r^{\mu}\left|z_{1}\right|^{m-\mu+\mu \beta(l)} \\
& >M\left|z_{1}\right|^{l} \geq\left|Q\left(z_{1}, z^{\prime}, z_{n}\right)\right| .
\end{aligned}
$$

Let now $\zeta \in B^{1}\left(0, \sigma\left|z_{1}\right|\right)$ with $\left(P_{m}+Q\right)\left(z_{1}, z^{\prime}, \zeta\right)=0$ be given. Then

$$
\left|P_{m}\left(z_{1}, z, \zeta\right)\right|=\left|Q\left(z_{1}, z^{\prime}, \zeta\right)\right| \leq M\left|z_{1}\right|^{l}
$$

and thus there is $l \leq d\left(z_{1}, z^{\prime}\right)$ with $\zeta \in D_{l}\left(z_{1}, z^{\prime}\right)$. Let $w \in D_{l}\left(z_{1}, z^{\prime}\right)$ be a zero of $F\left(z_{1}, z^{\prime}, \cdot\right)$. Then $|\zeta-w|<4 \delta$ since the sum of the radii of all disks $D_{l}\left(z_{1}, z^{\prime}\right)$ is at most $2 \delta$. We have shown that the estimate in (a) holds. To see that $|w|<\sigma\left|z_{1}\right|$, note that $F\left(z_{1}, z^{\prime}, w\right)=0$ implies the existence of $j$, $1 \leq j \leq \mu$, such that $w=\beta_{j}\left(z_{1}, z^{\prime}\right)$. Hence the choice of $\eta$ implies

$$
|w| \leq\left|z_{1}\right|\left|\beta_{j}\left(1, z^{\prime} /\left|z_{1}\right|\right)\right| \leq \frac{\sigma\left|z_{1}\right|}{2} .
$$

Thus the proof of Part (a) is complete.

To prove (b) we note first that under the present hypothesis we can choose $\eta$ and $\sigma$ so small that $B^{1}(1, \eta) \times B^{n-2}(0, \eta) \times B^{1}(0, \sigma)$ is contained in the set $U$ which exists by local hyperbolicity. This implies that the zeros $\beta_{j}\left(z^{\prime}, z_{n}\right)$ are all real whenever $\left(z^{\prime}, z_{n}\right) \in \Gamma^{\prime}$ is real. Hence the estimate in (a) implies the one in (b).

Lemma 3.7. Let $V$ be an algebraic variety in $\mathbb{C}^{n}$ and $\omega$ a weight function. Assume that for $\xi=(1,0, \ldots, 0)$ and $G=B^{n-1}(0, \delta) \times B^{1}(0, \sigma)(0<\delta, \sigma \leq$ 1) the map $\pi: V \cap \Gamma(\xi, G, r) \rightarrow \Gamma(\xi, G, r), \pi\left(z^{\prime}, z_{n}\right):=\left(z^{\prime}, 0\right)$, is proper and satisfies the following condition:

$$
\begin{aligned}
& \text { There exists } C>0 \text { such that }\left|\operatorname{Im} z_{n}\right| \leq C \omega(z) \\
& \text { for each } z \in V \cap \Gamma(\xi, G, r) \text { with } \pi(z) \text { real. }
\end{aligned}
$$

Then $V$ satisfies $\operatorname{PL}(V, \Gamma(\xi, G, r), \omega)$.

Proof. To show that there are a compact set $K \subset G$ and a constant $A_{1} \geq 1$ such that $V$ satisfies $\operatorname{PL}(V, \Gamma(\xi, G, r), \omega)$ let $K:=\frac{1}{2} \bar{G}$, fix $u \in \operatorname{PSH}(V \cap$ $\Gamma(\xi, G, R))$, and assume that $u$ satisfies Conditions $3.1(\alpha)$ and $(\beta)$. Then 
let $G^{\prime}:=B^{n-1}(0, \delta)$, fix $t>r$, and define

$$
\begin{aligned}
\varphi: G^{\prime} & \rightarrow[-\infty, \infty[ \\
z^{\prime} & \mapsto \max \left\{u(t \xi+t z): t \xi+t z \in V, z \in G, \pi(z)=\left(z^{\prime}, 0\right)\right\} .
\end{aligned}
$$

Then $\varphi$ is plurisubharmonic outside the branch locus of $\pi$. Since $\pi$ is proper by hypothesis, it follows from Hörmander [8], Lemma 4.4, that $\varphi$ extends to a plurisubharmonic function on $G^{\prime}$. Condition $3.1(\alpha)$ for $u$ and $0<\delta$, $\sigma \leq 1$ imply

$$
\varphi\left(z^{\prime}\right) \leq \max \{|t(\xi+z)|: z \in G\} \leq 2 t,
$$

while Condition $3.1(\beta)$ together with (3.6) implies

$$
\begin{aligned}
\varphi\left(z^{\prime}\right) & \leq \max \left\{\rho|\operatorname{Im}(t \xi+t z)|: t \xi+t z \in V, z \in G, \pi(z)=\left(z^{\prime}, 0\right)\right\} \\
& \leq \max \{\rho C \omega(t z): z \in G\} \leq \rho C \omega(t) .
\end{aligned}
$$

From these two estimates for $\varphi$ and classical estimates of the harmonic measure of the half disk (see, e.g., Nevanlinna [16], Section 38) it now follows that there is a constant $A_{0}$, depending only on the dimension, so that

$$
\varphi\left(z^{\prime}\right) \leq \frac{A_{0}}{\delta} 2 t\left|\operatorname{Im} z^{\prime}\right|+\rho C \omega(t), z \in \overline{B^{n-1}(0, \delta / 2)} .
$$

To evaluate this further, note that for $k \in K$ we have

$$
|t \xi+t k| \geq t(1-|k|) \geq \frac{t}{2} .
$$

Note also that our requirements on the weight functions imply the existence of a constant $L>0$ such that $\omega(2 s) \leq L \omega(s)$ for $s \geq 0$. Therefore, the definition of $\varphi$ and the previous estimates imply for $t \xi+t k \in V$

$$
\begin{aligned}
u(t \xi+t k) \leq \varphi(k) & \leq \frac{2 A_{0}}{\delta} t|\operatorname{Im} k|+\rho C \omega(t) \\
& \leq \frac{2 A_{0}}{\delta}|\operatorname{Im}(t \xi+t k)|+\rho C L \omega(t \xi+t k) .
\end{aligned}
$$

Since $k \in K$ and $t>r$ were chosen arbitrarily, this estimate shows that $u$ satisfies $3.1(\gamma)$ for $A_{1}:=\frac{2 A_{0}}{\delta}$ and $B_{\rho}:=\rho C L$.

Theorem 3.8. Let $P_{m} \in \mathbb{C}\left[z_{1}, \ldots, z_{n}\right]$ be homogeneous of degree $m \geq 2$ and assume that $V\left(P_{m}\right)$ satisfies $\mathrm{PL}\left(\mathbb{R}^{n}, \omega_{0}\right)$. Let

$$
\nu:=\max \left\{\operatorname{deg}\left(P_{m}\right)_{\theta}: \theta \in V\left(P_{m}\right) \cap S^{n-1}\right\}
$$

and define

$$
\beta(l):=\max \left(0,1-\frac{m-l}{\nu}\right) \quad \text { for } 0 \leq l<m .
$$

If for each $\xi \in V\left(P_{m}\right)_{\operatorname{sing}} \cap S^{n-1}$ the variety $V\left(P_{m}\right)$ is locally hyperbolic at $\xi$, then for each $Q \in \mathbb{C}\left[z_{1}, \ldots, z_{n}\right]$ with $\operatorname{deg} Q \leq l<m$ the variety $V\left(P_{m}+Q\right)$ satisfies $\mathrm{PL}\left(\mathbb{R}^{n}, \omega_{\beta(l)}\right)$, where $\omega_{\beta}$ is defined in Example 2.2 . 
Proof. Since $V\left(P_{m}\right)$ satisfies $\mathrm{PL}\left(\mathbb{R}^{n}, \omega_{0}\right)$ by hypothesis, the theorem follows from Proposition 3.3 once we show that the second condition in 3.3 (b) is fulfilled. To show this, we first factorize $P_{m}=\prod_{j=1}^{s} q_{j}^{k_{j}}$, where the polynomials $q_{j}$ are irreducible and where $\prod_{j=1}^{s} q_{j}$ is square-free. Since $V\left(P_{m}\right)$ satisfies $\operatorname{PL}\left(\mathbb{R}^{n}, \omega_{0}\right)$, also $V\left(q_{j}\right)$ has this property for each $j$. By Meise, Taylor, and Vogt [14], Lemma 2, this implies that there exists $c_{j} \in \mathbb{C},\left|c_{j}\right|=1$, so that $c_{j} q_{j}$ has real coefficients. Hence it is no restriction to assume that each $q_{j}$ has real coefficients.

Now fix a regular point $a \in V\left(P_{m}\right)$ of length 1 . Then there exists an index $i$ so that $q_{i}(a)=0$. This implies $q_{j}(a) \neq 0$ for all $j \neq i$ by the following argument: If $q_{j}(a)=0$ for some $j \neq i$ then $V\left(q_{j}\right)$ and $V\left(q_{i}\right)$ must coincide in a neighborhood of $a$ since $a$ is a regular point of $V\left(P_{m}\right)$. But then $V\left(q_{j}\right)=V\left(q_{i}\right)$ since both varieties are irreducible. Hence $q_{j}$ and $q_{i}$ are proportional, in contradiction to $\prod_{l=1}^{s} q_{l}$ being square-free.

Next note that

$$
\left(P_{m}\right)_{a}=\left(\left(q_{i}\right)_{a}\right)^{k_{i}} \prod_{j \neq i}\left(q_{j}(a)\right)^{k_{j}} .
$$

Since $a$ is a regular point of $V\left(P_{m}\right)$ and hence of $V\left(q_{i}\right)$, the localization satisfies $\left(q_{i}\right)_{a}(z)=\sum_{j=1}^{n} \frac{\partial q_{i}}{\partial z_{j}}(a) z_{j}$, which implies $\operatorname{deg}\left(P_{m}\right)_{a}=k_{i} \leq \nu$. After a real linear change of variables we may assume $a=(1,0, \ldots, 0)$ and $\frac{\partial q_{i}}{\partial z_{n}}(a) \neq$ 0 . Then the real and the complex implicit function theorem imply the existence of a neighborhood $U$ of $(1,0, \ldots, 0) \in \mathbb{C}^{n-1}$, of $\delta>0$, and of a holomorphic function $\beta: U \rightarrow B^{1}(0, \delta)$ which is real over real points so that

$$
V\left(P_{m}\right) \cap(U \times W)=\left\{\left(z^{\prime}, \beta\left(z^{\prime}\right)\right): z^{\prime} \in U\right\} .
$$

Hence $V\left(P_{m}\right)$ is locally hyperbolic at $a$ with respect to $\pi\left(z^{\prime}, z_{n}\right):=\left(z^{\prime}, 0\right)$ in these coordinates.

If $a \in V\left(P_{m}\right) \cap S^{n-1}$ is a singular point of $V\left(P_{m}\right)$, then $V\left(P_{m}\right)$ is locally hyperbolic at $a$ by hypothesis. Then we can perform a real linear change of coordinates so that in the new coordinates $a=(1,0, \ldots, 0)$ and $\pi:\left(z^{\prime}, z_{n}\right) \mapsto$ $\left(z^{\prime}, 0\right)$ is the projection which exists by local hyperbolicity. If we let $\mu=$ $\operatorname{deg}\left(P_{m}\right)_{a}$, then in both cases the hypotheses of Lemma 3.6 (b) are fulfilled. Now Lemma 3.6 implies that the hypotheses of Lemma 3.7 are fulfilled in a suitable cone $\Gamma\left(a, G_{a}, r_{a}\right)$ for $\omega=\omega_{\beta(l, a)}$, where $\beta(l, a)=\max \left(0,1-\frac{m-l}{\mu}\right)$. By the definition of $\mu$ we have $\mu \leq \nu$ and hence $\beta(l, a) \leq \beta(l)$. Thus, the second condition of Proposition 3.3 (b) holds with $\omega=\omega_{\beta(l)}$, which completes the proof of the theorem.

Remark. Theorem 3.8 also holds if we replace the hypothesis " $V\left(P_{m}\right)$ satisfies $\mathrm{PL}\left(\mathbb{R}^{n}, \omega_{0}\right)$ " by the following one: "Each irreducible factor of $P_{m}$ has real coefficients up to a complex factor and is not elliptic". Under this hypothesis, the present proof shows that $V\left(P_{m}\right)$ is locally hyperbolic at each 
real regular point of $V\left(P_{m}\right) \cap S^{n-1}$. Hence the hypotheses imply that this property holds at each point of $V\left(P_{m}\right) \cap S^{n-1}$. By Hörmander [8], Theorem 6.5, this implies that $V\left(P_{m}\right)$ satisfies Condition (HPL) and therefore it follows from Meise, Taylor, and Vogt [15], Corollary 3.14 that $V\left(P_{m}\right)$ satisfies $\operatorname{PL}\left(\mathbb{R}^{n}, \omega_{0}\right)$, which is needed for the application of Proposition 3.3. Otherwise the proof remains unchanged.

Remark. Note that for $n \geq 4$ there are homogeneous polynomials $P_{m} \in$ $\mathbb{C}\left[z_{1}, \ldots, z_{n}\right]$ for which $V\left(P_{m}\right)$ satisfies $\operatorname{PL}\left(\mathbb{R}^{n}, \omega_{0}\right)$ but which are not locally hyperbolic at some singular points of $V\left(P_{m}\right) \cap S^{n-1}$. When $n=3$, this cannot happen, as a result of Hörmander [8] shows. This fact will be used in Corollary 3.12 below.

As a corollary of Theorem 3.8 we get:

Corollary 3.9. Let $k_{j} \in \mathbb{N}$ and $P_{j} \in \mathbb{R}\left[z_{1}, \ldots, z_{n}\right], 1 \leq j \leq s$, be given. Assume that each $P_{j}$ is irreducible, homogeneous of degree $q_{j}$, and not elliptic and that $\prod_{j=1}^{s} P_{j}$ is square-free. Set $m:=\sum_{j=1}^{s} q_{j} k_{j}, P:=\prod_{j=1}^{s} P_{j}^{k_{j}}$, $k:=\max _{1 \leq j \leq s} k_{j}$, assume $m \geq 2$, and let $\beta(l)$ be as in (3.7) with $\nu=k$. If all points in $V(P) \cap S^{n-1}$ are regular points of $V(P)$, then for each $Q \in$ $\mathbb{C}\left[z_{1}, \ldots, z_{n}\right]$ with $\operatorname{deg} Q \leq l$ the variety $V(P+Q)$ satisfies $\operatorname{PL}\left(\mathbb{R}^{n}, \omega_{\beta(l)}\right)$.

Proof. Since the localization of a product equals the product of the localizations of its factors, the present hypotheses imply

$$
\nu=\max \left\{\operatorname{deg}(P)_{\theta}: \theta \in V\left(P_{m}\right) \cap S^{n-1}\right\}=\max _{j=1, \ldots, s} k_{j}=k .
$$

Since all points of $V(P) \cap S^{n-1}$ are regular points of $V(P)$, the corollary follows from Theorem 3.8.

As an obvious consequence of Corollary 3.9 we get the following result which is a reformulation of [4], Corollary 4.7:

Corollary 3.10. Let $P \in \mathbb{R}\left[z_{1}, \ldots, z_{n}\right]$ be homogeneous of degree $\mu$ and assume that $\operatorname{grad} P(x) \neq 0$ for all $x \in V(P) \cap S^{n-1}$. Let $k \in \mathbb{N}$ be given so that $k \mu \geq 2$. Then for each $Q \in \mathbb{C}\left[z_{1}, \ldots, z_{n}\right]$ with $\operatorname{deg} Q=: l<k \mu$ the variety $V\left(P^{k}+Q\right)$ satisfies $\mathrm{PL}\left(\mathbb{R}^{n}, \omega_{\beta(l)}\right)$ for $\beta(l)$ as in (3.7).

It will be shown in Theorem 3.14 that in Corollary 3.10 the condition $\operatorname{grad} P(x) \neq 0$ for all $x \in V(P) \cap S^{n-1}$ is in fact necessary. To prove this result, we use the following lemma:

Lemma 3.11. Let $P \in \mathbb{R}\left[z_{1}, \ldots, z_{n}\right]$ be homogeneous of degree $m \geq 2$, let

$$
\nu:=\max \left\{\operatorname{deg} P_{\theta}: \theta \in V(P) \cap S^{n-1}\right\},
$$

and fix $p \in \mathbb{N}$ with $1 \leq p<\nu$ and a weight function $\omega$. If for each $Q \in$ $\mathbb{C}\left[z_{1}, \ldots, z_{n}\right]$ with $\operatorname{deg} Q \leq m-\nu+p$ the variety $V(P+Q)$ satisfies $\mathrm{PL}\left(\mathbb{R}^{n}, \omega\right)$, then $t^{p / \nu}=O(\omega(t))$ as $t$ tends to infinity. 
Proof. Fix $\xi \in S^{n-1}$ with $\operatorname{deg} P_{\xi}=\nu$. After a real linear change of variables we may assume $\xi=(0, \ldots, 0,1)$. Hence [3], Lemma 3.9, implies

$$
P\left(z^{\prime}, z_{n}\right)=\sum_{j=\nu}^{m} z_{n}^{m-j} Q_{j}\left(z^{\prime}\right),
$$

where the polynomials $Q_{j} \in \mathbb{C}\left[z_{1}, \ldots, z_{n-1}\right]$ are either zero or homogeneous of degree $j$ and where $Q_{\nu}\left(z^{\prime}\right)=P_{\xi}\left(z^{\prime}, z_{n}\right)$. Now let $S:=P+i z_{n}^{m-\nu+p}$ and note that $V(S)$ satisfies $\mathrm{PL}\left(\mathbb{R}^{n}, \omega\right)$ by the present hypothesis. To apply Lemma 2.8 , let $d:=(p, \ldots, p, \nu)$. Then

$$
q\left(z^{\prime}, z_{n}\right):=z_{n}^{m-\nu} Q_{\nu}\left(z^{\prime}\right)+i z_{n}^{m-\nu+p}
$$

has $d$-degree $(m-\nu+p) \nu$. For $\nu+1 \leq j \leq m$ the term $z_{n}^{m-j} Q_{j}\left(z^{\prime}\right)$ has $d$-degree $(m-j) \nu+j p$. Since

$$
(m-\nu+p) \nu-((m-j) \nu+j p)=(j-\nu)(\nu-p)>0
$$

the polynomial $q$ is the term in $S$ with the highest $d$-degree. Next choose $a \in S^{n-1}$ such that $Q_{\nu}(a) \neq 0$ and consider the polynomial

$$
\lambda \mapsto q(\lambda a, 1)=Q_{\nu}(\lambda a)+i=\lambda^{\nu} Q_{\nu}(a)+i .
$$

Since $Q_{\nu}$ has real coefficients by hypothesis, we can choose $\lambda_{0} \in \mathbb{C} \backslash \mathbb{R}$ such that $\zeta^{\prime}:=\lambda_{0} a \in \mathbb{C}^{n-1} \backslash \mathbb{R}^{n-1}$ satisfies $q\left(\zeta^{\prime}, 1\right)=0$. Finally, note that the equation

$$
0=q\left(z^{\prime}, 1\right)=Q_{\nu}\left(z^{\prime}\right)+i
$$

has no real solutions since $Q_{\nu}$ has real coefficients. Thus we have shown that all conditions of Lemma 2.8 are fulfilled. Therefore, the present lemma follows from Lemma 2.8 .

Corollary 3.12. Let $P_{m} \in \mathbb{C}[x, y, z]$ be homogeneous of degree $m \geq 2$, let

$$
\nu:=\max \left\{\operatorname{deg}\left(P_{m}\right)_{\theta}: \theta \in V\left(P_{m}\right) \cap S^{2}\right\}
$$

and define $\beta(l)$ as in (3.7). Then the following assertions are equivalent:

(a) $V\left(P_{m}\right)$ satisfies $\operatorname{PL}\left(\mathbb{R}^{3}, \omega_{0}\right)$.

(b) For each $0 \leq l<m$ and for each $Q \in \mathbb{C}[x, y, z] \operatorname{deg} Q \leq l$, the variety $V\left(P_{m}+Q\right)$ satisfies $\mathrm{PL}\left(\mathbb{R}^{3}, \omega_{\beta(l)}\right)$.

(c) There exist $Q \in \mathbb{C}[x, y, z], \operatorname{deg} Q<m$, and a weight function $\omega$ such that the variety $V\left(P_{m}+Q\right)$ satisfies $\mathrm{PL}\left(\mathbb{R}^{3}, \omega\right)$.

Moreover, the numbers $\beta(l)$ are optimal in the following sense: If $l$ satisfies $m-\nu \leq l<m$ and if for some weight function $\omega$ and each polynomial $Q$ of degree at most $l$ the variety $V(P+Q)$ satisfies $\mathrm{PL}\left(\mathbb{R}^{3}, \omega\right)$, then $t^{\beta(l)}=$ $O(\omega(t))$ as $t$ tends to infinity.

Proof. (b) $\Rightarrow$ (c): This holds obviously.

$(\mathrm{c}) \Rightarrow(\mathrm{a})$ : This holds by Meise, Taylor, and Vogt [15], Theorem 4.1. 
(a) $\Rightarrow(\mathrm{b})$ : Since $V\left(P_{m}\right)$ satisfies $\operatorname{PL}\left(\mathbb{R}^{3}, \omega_{0}\right)$ it also satisfies the PhragménLindelöf condition $\operatorname{HPL}\left(\mathbb{R}^{3}\right)$, considered in Hörmander [8] (by Meise, Taylor, and Vogt [15], Proposition 3.9). By Hörmander [8], Theorem 6.5, this implies that $V\left(P_{m}\right)$ is locally hyperbolic at each $\xi \in V\left(P_{m}\right) \cap S^{2}$. Hence (b) follows from Theorem 3.8.

The additional assertion obviously follows from Lemma 3.11.

Remark. Note that Corrollary 3.12 implies Theorem 1.1 by the results of Meise, Taylor, and Vogt [13], mentioned in 2.5.

From Hörmander [7], 10.4.11, we recall the following definition:

Definition 3.13. A polynomial $P \in \mathbb{C}\left[z_{1}, \ldots, z_{n}\right]$ is said to be of principal type if its principal part $P_{m}$ satisfies

$$
\sum_{j=1}^{n}\left|\frac{\partial P_{m}}{\partial x_{j}}(x)\right| \neq 0 \quad \text { for each } x \in \mathbb{R}^{n} \backslash\{0\} .
$$

Note that by Euler's rule $\left\langle x, \operatorname{grad} P_{m}(x)\right\rangle=m P_{m}(x)$, so $P$ is of principal type if and only if $\operatorname{grad} P_{m}(x) \neq 0$ for each $x \in V\left(P_{m}\right) \cap \mathbb{R}^{n} \backslash\{0\}$.

Theorem 3.14. For a homogeneous polynomial $P \in \mathbb{C}\left[z_{1}, \ldots, z_{n}\right]$ of degree $\mu \geq 1$ and $k \in \mathbb{N}$ satisfying $\mu k \geq 2$ the following assertions are equivalent (for the definition of the weights $\omega_{\alpha}$ see Example 2.2):

(a) $V\left(P^{k}+Q\right)$ satisfies $\operatorname{PL}\left(\mathbb{R}^{n}, \omega_{0}\right)$ for each $Q \in \mathbb{C}\left[z_{1}, \ldots, z_{n}\right]$ with $\operatorname{deg} Q \leq$ $(\mu-1) k$.

(b) For each $p \in \mathbb{N}_{0}, 0 \leq p<k$, and each $Q \in \mathbb{C}\left[z_{1}, \ldots, z_{n}\right]$ with $\operatorname{deg} Q \leq$ $(\mu-1) k+p$ the variety $V\left(P^{k}+Q\right)$ satisfies $\operatorname{PL}\left(\mathbb{R}^{n}, \omega_{p / k}\right)$.

(c) There exists $p \in \mathbb{N}_{0}, 0 \leq p<k$, such that the assertion in (b) holds.

(d) $P$ is of principal type and real up to a complex factor, and each irreducible factor of $P$ admits a real zero $\xi \neq 0$.

Proof. From (d) we get (a) and (b) by Corollary 3.10. Obviously, (a) implies (c) and also (b) implies (c). Hence it suffices to prove that (c) implies (d). To do so, note first that $V\left(P^{k}\right)$ and hence $V(P)$ satisfies $\operatorname{PL}\left(\mathbb{R}^{n}, \omega_{p / k}\right)$. Since $P$ is homogeneous, it follows from Meise, Taylor, and Vogt [15], Theorem 3.3, that $V(P)$ satisfies $\operatorname{PL}\left(\mathbb{R}^{n}, \omega_{0}\right)$. From this and [15], Theorem 3.13 , we get that for each irreducible factor $q$ of $P$ we have $\operatorname{dim}_{\mathbb{R}} V(q) \cap \mathbb{R}^{n}=n-1$. Thus the last condition in (d) is fulfilled. Since $V(P)$ satisfies $\operatorname{PL}\left(\mathbb{R}^{n}, \omega_{0}\right)$, Lemma 2 in Meise, Taylor, and Vogt [14] implies the existence of $\lambda \in \mathbb{C} \backslash\{0\}$ such that $\lambda P$ has real coefficients. Hence the second condition in $(\mathrm{d})$ holds, and we may assume that $P$ has real coefficients. To show that $P$ is of principal type we argue by contradiction and assume that for some $a \in$ $V(P) \cap S^{n-1}$ we have $\operatorname{grad} P(a)=0$. This implies $\operatorname{deg} P_{a} \geq 2$. Since the localization of a product is the product of the localizations of its factors it follows that

$$
\nu:=\max \left\{\operatorname{deg}\left(P^{k}\right)_{\theta}: \theta \in V\left(P^{k}\right) \cap S^{n-1}\right\} \geq 2 k .
$$


Now let $s:=\nu-k+p$, where $0 \leq p<k$ is chosen according to (c). Then

$$
\frac{s}{\nu}=1-\nu^{-1}(k-p)>1-k^{-1}(k-p)=\frac{p}{k} \text { and } \mu k-\nu+s=(\mu-1) k+p .
$$

Hence (c) implies that for each $Q \in \mathbb{C}\left[z_{1}, \ldots, z_{n}\right]$ with $\operatorname{deg} Q \leq \mu k-\nu+s$ the variety $V\left(P^{k}+Q\right)$ satisfies $\operatorname{PL}\left(\mathbb{R}^{n}, \omega_{p / k}\right)$. By Lemma 3.11 this implies

$$
t^{s / \nu}=O\left(\omega_{p / k}(t)\right)=O\left(t^{p / k}\right) .
$$

Since $\frac{s}{\nu}>\frac{p}{k}$, this contradiction completes the proof.

Remark. Theorem 3.14 extends [4], Theorem 4.3 and Corollary 4.7.

\section{Further results and examples.}

In this section we first indicate that there are further variants of Lemma 3.6 which may be helpful in considering examples. Then we provide several examples to illustrate the results of the previous section and to explain the difficulties that one encounters in proving perturbation results.

Lemma 4.1. Let $P_{m} \in \mathbb{C}[x, y, z]$ be homogeneous of degree $m \geq 2$, let $\xi \in$ $V\left(P_{m}\right) \cap S^{2}$ satisfy $\operatorname{deg}\left(P_{m}\right)_{\xi}=: \mu \geq 2$, and assume that $V\left(P_{m}\right)$ is locally hyperbolic at $\xi$ and that $\left(P_{m}\right)_{\xi}$ is square-free. Let $Q \in \mathbb{C}[x, y, z]$ with $\operatorname{deg} Q<$ $m$ be given. Decompose $Q$ as $Q=\sum_{j=\mu}^{m-1} q_{j}$, where $q_{j}$ is either homogeneous of degree $j$ or zero. If $\operatorname{deg}\left(q_{j}\right)_{\xi} \geq \mu$ for each $j$, then $V\left(P_{m}+Q\right)$ satisfies $\operatorname{PL}\left(V\left(P_{m}+Q\right), \Gamma\left(\xi, G_{\xi}, r_{\xi}\right), \omega_{0}\right)$ in a suitable cone $\Gamma\left(\xi, G_{\xi}, r_{\xi}\right)$.

Proof. After a real linear change of variables we may assume $\xi=(1,0,0)$. By [3], Lemma 3.9, we then have in these coordinates

$$
P_{m}(x, y, z)=\sum_{j=\mu}^{m} x^{m-j} p_{j}(y, z),
$$

where $p_{j}$ is either homogeneous of degree $j$ or identically zero and where $p_{\mu}(y, z)=\left(P_{m}\right)_{\xi}(x, y, z)$. We may also assume that the coordinates have been chosen so that $\pi(x, y, z):=(x, y, 0)$ is a projection for which the local hyperbolicity condition holds. Then we get

$$
p_{\mu}(y, z)=c \prod_{j=1}^{\mu}\left(z-a_{j} y\right)
$$

for suitable numbers $c, a_{1}, \ldots, a_{\mu} \in \mathbb{C}$. Since $p_{\mu}$ is square-free by hypothesis, we have $a_{i} \neq a_{j}$ for $i \neq j$ and hence

$$
\delta:=\min \left\{\left|a_{i}-a_{j}\right|: 1 \leq i, j \leq \mu, i \neq j\right\}>0 .
$$

By Braun [1], Corollary 12, the local hyperbolicity of $V\left(P_{m}\right)$ at $\xi$ implies the existence of $\sigma>0$ and $0<\eta<\frac{1}{2}$ and of holomorphic functions $\beta_{j}$ : $B(1, \eta) \times B(0, \eta) \rightarrow B(0, \sigma), 1 \leq j \leq \mu$ so that 


$$
\begin{aligned}
V\left(P_{m}\right) \cap(B(1, \eta) \times B(0, \eta) \times B(0, \sigma)) & \\
= & \bigcup_{j=1}^{\mu}\left\{\left(x, y, \beta_{j}(x, y)\right):(x, y) \in B(1, \eta) \times B(0, \eta)\right\}
\end{aligned}
$$

and so that $\beta_{j}(x, y)$ is real for real $(x, y)$. By the homogeneity of $P_{m}$ we get

$$
\beta_{j}(x, y)=x \beta_{j}\left(1, \frac{y}{x}\right)=x \sum_{k=1}^{\infty} b_{j, k}\left(\frac{y}{x}\right)^{k}, \quad 1 \leq j \leq \mu,
$$

where $\left\{b_{j, 1}: 1 \leq j \leq \mu\right\}=\left\{a_{j}: 1 \leq j \leq \mu\right\}$. Hence we may assume $a_{j}=b_{j, 1}$ for $1 \leq j \leq \mu$. It is no restriction to assume $\eta$ to be so small that

$$
\left|\sum_{k=2}^{\infty} b_{j, k}\left(\frac{y}{x}\right)^{k}\right| \leq \delta \quad \text { if }(x, y) \in B(1, \eta) \times B(0, \eta) .
$$

Arguing as in the proof of Lemma 3.6 we get for $\eta>0$ small enough with

$$
\Gamma:=\left\{(x, y, z) \in \mathbb{C}^{3}:\left|\frac{x}{|x|}-1\right|,|x|>R,\left|\frac{y}{x}\right|<\eta,\left|\frac{z}{x}\right|<\sigma\right\}
$$

and $\Gamma^{\prime}:=\left\{(x, y) \in \mathbb{C}^{3}:(x, y, 0) \in \Gamma\right\}$ that

$$
P_{m}(x, y, z)=U(x, y, z) F(x, y, z), \quad(x, y, z) \in \Gamma,
$$

where $F(x, y, z)=\prod_{j=1}^{\mu}\left(z-\beta_{j}(x, y)\right)$. We also get the existence of $\alpha>0$ such that

$$
|U(x, y, z)| \geq \alpha|x|^{m-\mu},(x, y, z) \in \Gamma .
$$

Choosing $\eta$ small enough, we get

$$
\left|\beta_{j}\left(1, \frac{y}{x}\right)-a_{j} \frac{y}{x}\right|=\left|\sum_{k=2}^{\infty} b_{j, k}\left(\frac{y}{x}\right)^{k}\right| \leq 2 \delta\left|\frac{y}{x}\right|, 1 \leq j \leq \mu .
$$

Next fix $1 \leq i \leq \mu$ and define for $(x, y) \in \Gamma^{\prime}$

$$
z(x, y, \lambda):=\beta_{i}(x, y)+\lambda
$$

where for some $\rho>1$ (to be determined later), $\lambda \in \mathbb{C}$ satisfies

$$
|\lambda|=\min (\rho, \delta|y|) \text {. }
$$

For $j \neq i$ the previous choices imply

$$
\begin{aligned}
\left|z(x, y, \lambda)-\beta_{j}(x, y)\right| & =\left|\left(a_{i}-a_{j}\right) y+y \sum_{k=2}^{\infty}\left(b_{i, k}-b_{j, k}\right)\left(\frac{y}{x}\right)^{k}+\lambda\right| \\
& \geq 4 \delta|y|-2 \delta|y|-|y|=\delta|y| .
\end{aligned}
$$

Moreover, we get 


$$
\begin{aligned}
\left|\frac{z(x, y, \lambda)}{x}\right| & =\left|a_{i} \frac{y}{x}+\sum_{k=2}^{\infty} b_{i, k}\left(\frac{y}{x}\right)^{k}+\frac{\lambda}{x}\right| \\
& \leq\left(\left|a_{i}\right|+2 \delta+\delta\right)\left|\frac{y}{x}\right| \\
& \leq \max _{1 \leq j \leq \mu}\left(\left|a_{j}\right|+3 \delta\right) \eta<\sigma,
\end{aligned}
$$

provided that $\eta$ is small enough. All together we get for $(x, y) \in \Gamma^{\prime}$

$$
\left|P_{m}(x, y, z(x, y, \lambda))\right|=|(U F)(x, y, z(x, y, \lambda))| \geq \alpha|x|^{m-\mu}|\lambda|(\delta|y|)^{\mu-1} .
$$

Now fix $Q=\sum_{j=\mu}^{m-1} q_{j}$ as in the hypothesis. For $\kappa_{j}:=\operatorname{deg}\left(q_{j}\right)_{\xi}$ there is $M_{j}$ with

$$
\left|q_{j}(1, \eta, \zeta)\right| \leq M_{j}|(\eta, \zeta)|^{\kappa_{j}} \quad \text { if }|(\eta, \zeta)| \leq 1
$$

Hence (4.1) implies

$$
\begin{aligned}
\left|q_{j}(x, y, z(x, y, \lambda))\right| & =\left|q_{j}\left(1, \frac{y}{x}, \frac{z(x, y, \lambda)}{x}\right)\right||x|^{j} \\
& \leq M_{j}\left(1+\left|a_{j}\right|+3 \delta\right)^{\kappa_{j}}\left|\frac{y}{x}\right|^{\kappa_{j}}|x|^{j} .
\end{aligned}
$$

Since $\kappa_{j} \geq \mu$ by hypothesis, the last estimate implies the existence of $M$ such that

$$
|Q(x, y, z(x, y, \lambda))| \leq M|x|^{m-1}\left|\frac{x}{y}\right|^{\mu}
$$

Now we claim that we can choose $R>1$ and $\rho>1$ so that

$$
M<\alpha|\lambda|\left|\frac{x}{y}\right| \delta^{\mu-1} \quad \text { for }|x|>R,(x, y) \in \Gamma^{\prime} .
$$

To see this, assume first $|\lambda|=\delta|y|$. Then

$$
\alpha|\lambda|\left|\frac{x}{y}\right| \delta^{\mu-1}=\alpha \delta^{\mu}|y|\left|\frac{x}{y}\right|=\alpha \delta^{\mu}|x|>M \quad \text { if }|x|>R=\max \left(1, \frac{M}{\delta^{\mu} \alpha}\right) .
$$

If $|\lambda|=\rho$ then

$$
\alpha|\lambda|\left|\frac{x}{y}\right| \delta^{\mu-1}=\gamma \rho\left|\frac{x}{y}\right| \delta^{\mu-1}>\alpha \rho \frac{1}{\eta} \delta^{\mu-1}>M
$$

if we choose $\rho>\frac{\eta M}{\alpha \delta^{\mu-1}}$. Hence (4.2) holds. From it and $\kappa_{j} \leq \mu$ we now get 


$$
\begin{aligned}
|Q(x, y, z(x, y, \lambda))| & \leq M|x|^{m-1}\left|\frac{y}{x}\right|^{\mu} \\
& <\left.\alpha|\lambda| \frac{x}{y}\left|\delta^{\mu-1}\right| x\right|^{m-1}\left|\frac{y}{x}\right|^{\mu} \\
& =\alpha|\lambda||x|^{m-1} \delta^{\mu-1}\left|\frac{y}{x}\right|^{\mu-1} \\
& \leq \alpha|\lambda||x|^{m-1} \delta^{\mu-1}\left|\frac{y}{x}\right|^{\mu-1} \\
& \leq\left|P_{m}(x, y, z(x, y, \lambda))\right|
\end{aligned}
$$

since $\left|\frac{y}{x}\right|<1$. From this estimate and the theorem of Rouché it follows for $(x, y) \in \Gamma^{\prime}, y \neq 0,1 \leq i \leq \mu$, that the functions $\zeta \mapsto P_{m}(x, y, \zeta)$ and $\zeta \mapsto$ $\left(P_{m}+Q\right)(x, y, \zeta)$ have the same number of zeros in the disk $\left|\zeta-\beta_{i}(x, y)\right|<r$, where $r=\min (\rho, \delta|y|)$. Since $P_{m}$ has exactly one zero in this disk, we get that $\left(P_{m}+Q\right)(x, y, \cdot)$ also has exactly one zero in that disk.

A similar application of the theorem of Rouché shows that for each $(x, y) \in$ $\Gamma^{\prime}$ the functions $\zeta \mapsto P_{m}(x, y, \zeta)$ and $\left(P_{m}+Q\right)(x, y, \zeta)$ have the same number of zeros in the disk of radius $\sigma|x|$. All together we have shown that under the present hypotheses the conclusion of Lemma 3.6 holds with $\beta(l)=0$. Since $\beta_{j}(x, y)$ is real for each $(x, y) \in \Gamma^{\prime}$ and $1 \leq j \leq \mu$, this implies the existence of a constant $C>0$ such that for each $(x, y, \zeta) \in \Gamma$ satisfying $\left(P_{m}+Q\right)(x, y, \zeta)=0$ we have the estimate

$$
|\operatorname{Im} \zeta| \leq C \text {. }
$$

Therefore, the assertion of the lemma follows from Lemma 3.6.

Lemma 4.1 does not hold without the hypothesis " $\left(P_{m}\right)_{\xi}$ is square-free". To provide an example for this fact, we will use the following lemma. Since its proof uses only basic calculus, we omit it.

Lemma 4.2. For $t>0$ and $a \in \mathbb{R}$ consider the polynomial

$$
p(z ; t, a):=\left(z^{2}-t^{2}\right)(z-2 t)+a .
$$

Then for each $t>0$ and each a satisfying $|a| \leq \frac{1}{2} t^{3}$, all zeros of $p(\cdot ; t, a)$ are real.

Example 4.3. Define $P_{6}, Q \in \mathbb{R}[x, y, z]$ by

$$
P_{6}(x, y, z):=\left(x z-y^{2}\right)\left(x z+y^{2}\right)\left(x z-2 y^{2}\right), \quad Q(x, y, z):=x^{2} y^{3} .
$$

Then the following assertions hold:

(a) For $\xi=( \pm 1,0,0)$, the variety $V\left(P_{6}+Q\right)$ satisfies $\operatorname{PL}\left(V\left(P_{6}+Q\right)\right.$, $\left.\Gamma\left(\xi, G_{\xi}, r_{\xi}\right), \omega_{1 / 3}\right)$.

(b) For each $\xi \in V\left(P_{6}\right) \cap S^{2}$, the variety $\xi \neq( \pm 1,0,0), V\left(P_{6}+Q\right)$ satisfies $\operatorname{PL}\left(V\left(P_{6}+Q\right), \Gamma\left(\xi, G_{\xi}, r_{\xi}\right), \omega_{0}\right)$.

(c) $V\left(P_{6}+Q\right)$ satisfies $\mathrm{PL}\left(\mathbb{R}^{3}, \omega_{1 / 3}\right)$. 
(d) If $V\left(P_{6}+Q\right)$ satisfies $\operatorname{PL}\left(\mathbb{R}^{3}, \omega\right)$ for some weight function $\omega$ then $t^{1 / 3}=$ $O(\omega(t))$ as $t$ tends to infinity.

Proof. (a) Let $\xi:=(1,0,0)$, define

$$
\Gamma:=\left\{(x, y, z) \in \mathbb{C}^{3}:\left|\frac{x}{|x|}-1\right|<\frac{1}{4},|y|<\frac{1}{4}|x|,|z|<\frac{1}{4}|x|,|x|>1\right\},
$$

and let $\Gamma^{\prime}=\left\{(x, y) \in \mathbb{C}^{2}:(x, y, 0) \in \Gamma\right\}$. Fix $(x, y) \in \Gamma^{\prime} \cap \mathbb{R}^{2}$ and assume first that $|y|>(2 x)^{2 / 3}$. Then we have

$$
\left|\frac{y^{3}}{x}\right|<\frac{1}{2}\left|\frac{y^{2}}{x}\right|^{3}
$$

Hence Lemma 4.2 implies that all zeros of the equation

$$
\left(P_{6}+Q\right)(x, y, z)=x^{3}\left(\left(z^{2}-\left(\frac{y^{2}}{x}\right)^{2}\right)\left(z-2 \frac{y^{2}}{x}\right)+\frac{y^{3}}{x}\right)
$$

are real.

Assume next that $(x, y) \in \Gamma^{\prime} \cap \mathbb{R}^{2}$ satisfies $|y|<2|x|^{2 / 3}$. If $y=0$ then obviously, $\left(P_{6}+Q\right)(x, 0, z)$ has a zero of order 3 at the origin. Hence we may assume $|y|>0$. Then let $\delta:=2^{1 / 3} e|y||x|^{-1 / 3}$ and apply the Lemma of Boutroux-Cartan to get

$$
\left|\left(z^{2}-\left(\frac{y^{2}}{x}\right)^{2}\right)\left(z-2 \frac{y^{2}}{x}\right)\right| \geq\left(\frac{\delta}{e}\right)^{3}=2\left|\frac{y^{3}}{x}\right|>\left|\frac{y^{3}}{x}\right|
$$

outside a finite number of disks for which the sum of their radii is at most $2 \delta$. Applying the theorem of Rouché at the boundary of these disks we get that for each zero $\zeta$ of the equation $\left(P_{6}+Q\right)(x, y, \zeta)=0$ we have

$$
|\operatorname{Im} \zeta| \leq 4 e 2^{1 / 3}\left|\frac{y^{3}}{x}\right|^{1 / 3} \leq 4 e 2^{1 / 3}\left(\frac{2^{3} x^{2}}{x}\right)^{1 / 3} \leq e 2^{4}|x|^{1 / 3} .
$$

Combining this estimate with Lemma 3.7, we get (a) for $\xi$. Since it is easy to check that the same arguments apply also for $-\xi$, the proof of (a) is complete.

(b) Whenever $\xi \in V\left(P_{6}\right) \cap S^{2}$ and $\xi \neq( \pm 1,0,0)$ and $\xi \neq(0,0, \pm 1)$ then $\operatorname{grad} P_{6}(\xi) \neq 0$. Hence a real linear change of coordinates shows that we can apply Lemma 3.6 with $\mu=1$. Hence in this case the assertion follows from Lemma 3.6 and 3.7. If $\xi=(0,0,1)$ then let

$$
\Gamma^{\prime}:=\left\{(y, z) \in \mathbb{C}^{2}:\left|\frac{z}{|z|}-1\right|<\frac{1}{4}, \quad|z|>2,|y|<\frac{1}{4}|z|\right\}
$$

and note that

$$
\left(P_{6}+Q\right)(x, 0, z)=x^{3} z^{3}
$$


has a zero of order 3 at the origin for each $|z|>0$. For $(y, z) \in \Gamma^{\prime} \cap \mathbb{R}^{2}$ with $y \neq 0$ we have

$$
\begin{array}{r}
\left(P_{6}+Q\right)(0, y, z)=2 y^{6}>0, \\
\left(P_{6}+Q\right)\left(\frac{3}{2} \frac{y^{2}}{z}, y, z\right)=-\frac{5}{8} y^{6}+\frac{9}{4} \frac{y^{7}}{z^{2}}=-y^{6}\left(\frac{5}{8}-\frac{9}{4} \frac{y}{z^{2}}\right)<0, \\
\left(P_{6}+Q\right)\left(-2 \frac{y^{2}}{z}, y, z\right)=-12 y^{6}+4 \frac{y^{7}}{z^{2}}=-y^{6}\left(12+\frac{4 y}{z^{2}}\right)<0 .
\end{array}
$$

Since $\left(P_{6}+Q\right)(\cdot, y, z)$ has degree three and real coefficients, this implies that for each $(y, z) \in \Gamma^{\prime} \cap \mathbb{R}^{2}$, all zeros of $x \mapsto\left(P_{6}+Q\right)(x, y, z)$ are real. Hence Lemma 3.7 implies the assertion of (b) also in this case. The same arguments apply for $\xi=(0,0,-1)$.

(c) This assertion follows from Proposition 3.3 since the second condition in 3.3 (b) holds by the present parts (a) and (b) and since $V\left(P_{6}\right)$ satisfies $\operatorname{PL}\left(\mathbb{R}^{3}, \omega_{0}\right)$. The latter assertion follows from the fact that $P_{6}$ is the product of three polynomials, each of which defines a wave operator.

(d) It is easy to check that $P_{6}+Q$ is $(3,2,1)$-quasihomogeneous and that the polynomial $z^{3}-2 z^{2}-z+3$ has a zero $\tau$ which is not real. Then $\zeta:=(1,1, \tau)$ satisfies

$$
\left(P_{6}+Q\right)(\zeta)=(\tau-1)(\tau+1)(\tau-2)+1=\tau^{3}-2 \tau^{2}-\tau+3=0 .
$$

Hence $P:=P_{6}+Q, \zeta$ and $d=(3,2,1)$ satisfy the hypotheses of [4], Lemma 3.2. Hence $(d)$ follows from this lemma.

The following example shows how Lemma 4.1 can be applied.

Example 4.4. Define the polynomial $P_{5}$ by

$$
P_{5}(x, y, z):=z^{2} y\left(x^{2}-y^{2}\right)+x^{5}+(x-y)^{2} x^{3}+y^{5} .
$$

Let $q_{3}, q_{4}$, and $p_{3}$ be polynomials in $\mathbb{C}[x, y]$, each of which is homogeneous of degree 3,4 , and 3 respectively or identically zero, and define

$$
Q(x, y, z):=q_{3}(x, y)+q_{4}(x, y)+z p_{3}(x, y) .
$$

Then $V\left(P_{5}+Q\right)$ satisfies $\operatorname{PL}\left(\mathbb{R}^{3}, \omega_{0}\right)$.

An interesting example of a perturbation $Q$ satisfying the above conditions is given by $Q(x, y, z):=x^{4}-x y^{2}$.

Proof. To derive the assertion from Proposition 3.3, let $P:=P_{5}+Q$, so that $P_{5}$ is the principal part of $P$. Some computation shows that $\operatorname{grad} P_{5}$ vanishes only on $V\left(P_{5}\right) \cap\{(0,0, t): t \in \mathbb{C}\}$ so that

$$
V\left(P_{5}\right)_{\text {sing }} \cap S^{2}=\{(0,0,1),(0,0,-1)\} .
$$

Since $P_{5}$ is irreducible, it follows from Meise, Taylor, and Vogt [15], Corollary 3.14 , that $V\left(P_{5}\right)$ satisfies $\mathrm{PL}\left(\mathbb{R}^{3}, \omega_{0}\right)$ if and only if $V\left(P_{5}\right)$ satisfies 
Hörmander's Phragmén-Lindelöf condition, which by Hörmander [8], Theorem 6.5 , is equivalent to $V\left(P_{5}\right)$ being locally hyperbolic at each $\xi \in V\left(P_{5}\right) \cap$ $S^{2}$. Since $P_{5}$ has real coefficients, this condition obviously holds at each regular point $\xi \in V\left(P_{5}\right) \cap S^{2}$. At the singular points $\xi_{ \pm}:=(0,0, \pm 1)$, it holds by the following observation: The reduction of $P_{5}$ at $\xi_{ \pm}$, defined by

$$
q_{ \pm}(x, y):=P_{5}(x, y, \pm 1)=y\left(x^{2}-y^{2}\right)+x^{5}+\left(x^{2}-y^{2}\right) x^{3}+y^{5},
$$

has a zero variety which is locally hyperbolic at the origin. Therefore it follows from [5], Lemma 6.1, that $V\left(P_{5}\right)$ satisfies $\mathrm{PL}_{\text {loc }}(\xi)$ and hence $V\left(P_{5}\right)$ is locally hyperbolic at $\xi_{ \pm}$, by Braun [1], Corollary 12 . Thus we have shown that $V\left(P_{5}\right)$ satisfies $\mathrm{PL}\left(\omega_{0}\right)$, i.e., the first condition of Proposition 3.3 (b) holds. To show that also the second one is fulfilled, note that $V(P)$ satisfies $\mathrm{PL}\left(V(P), \Gamma\left(\xi, G_{\xi}, r_{\xi}\right), \omega_{0}\right)$ for each $\xi \in V\left(P_{5}\right) \cap S^{2} \backslash\{ \pm \xi\}$ and a suitable cone $\Gamma\left(\xi, G_{\xi}, r_{\xi}\right)$ because of Lemma 3.6 and Lemma 3.7, since $\operatorname{grad} P_{5}(\xi)$ is not zero. To show that the same condition also holds at $\xi=\xi_{ \pm}$, note that $\operatorname{deg}\left(P_{5}\right)_{\xi_{ \pm}}=3$ and that $\left(P_{5}\right)_{\xi_{ \pm}}(x, y, z)=y\left(x^{2}-y^{2}\right)$ is square-free. The decomposition of $Q$ into homogeneous components is $Q=Q_{3}+Q_{4}$ with $Q_{3}(x, y, z)=q_{3}(x, y)$ and $Q_{4}(x, y, z)=q_{4}(x, y)+$ $z p_{3}(x, y)$. Hence $\operatorname{deg}\left(Q_{j}\right)_{\xi_{ \pm}} \geq 3=\operatorname{deg}\left(P_{5}\right)_{\xi_{ \pm}}$for $j=3,4$, and it follows from Lemma 4.1 that $V(P)$ satisfies $\operatorname{PL}\left(V(P), \Gamma\left(\xi_{ \pm}, G_{\xi_{ \pm}}, r_{\xi_{ \pm}}\right), \omega_{0}\right)$ for suitable cones $\Gamma\left(\xi_{ \pm}, G_{\xi_{ \pm}}, r_{\xi_{ \pm}}\right)$. This shows that also the second condition of 3.3 (b) is fulfilled. Therefore, the assertion follows from Proposition 3.3.

Example 4.5. Let $P \in \mathbb{R}[x, y, z]$ be defined as

$$
P(x, y, z):=z^{2}\left(x^{2}+y^{2}-z^{2}\right)^{3} .
$$

Then for each $Q \in \mathbb{C}[x, y, z]$ with $\operatorname{deg} Q \leq 5$ the operator $(P+Q)(D)$ admits a continuous linear right inverse on $\mathcal{D}^{\prime}\left(\mathbb{R}^{3}\right)$. If $\operatorname{deg} Q=6$ or $\operatorname{deg} Q=7$, then $(P+Q)(D)$ admits a continuous linear right inverse on $\mathcal{D}_{\left(\omega_{1 / 3}\right)}^{\prime}\left(\mathbb{R}^{3}\right)$ or $\mathcal{D}_{\left(\omega_{2 / 3}\right)}^{\prime}\left(\mathbb{R}^{3}\right)$, respectively. This follows from 2.5 and Proposition 3.9.

Remark. In Lemma 3.11 and Theorem 3.14 the statements are optimal if perturbations by arbitrary polynomials of a given degree are considered. For an individual polynomial it may happen that $(P+Q)(D)$ admits a continuous linear right inverse on $\mathcal{D}_{(\sigma)}^{\prime}\left(\mathbb{R}^{n}\right)$ for a weight function $\sigma$ which grows more slowly than indicated by 3.9 or 3.14 . Such examples can be constructed easily from our results, as we show next.

Example 4.6. Let $P(x, y, z):=\left(x^{2}+y^{2}-z^{2}\right)^{2}$. Then 2.5 and Theorem 3.14 imply that $(P+Q)(D)$ admits a continuous linear right inverse on $\mathcal{D}_{\left(\omega_{1 / 2}\right)}^{\prime}\left(\mathbb{R}^{3}\right)$ whenever $\operatorname{deg} Q \leq 3$ and that for each $\omega$ satisfying $\omega(t)=o\left(t^{1 / 2}\right)$ there is a polynomial $Q$ of degree 3 such that $(P+Q)(D)$ does not admit a continuous linear right inverse on $\mathcal{D}_{(\omega)}^{\prime}\left(\mathbb{R}^{3}\right)$. 
Nevertheless, in special cases like, e.g., $Q_{0}(x, y, z):=\left(x^{2}+y^{2}-z^{2}\right) y$, more can be said. For this example

$$
P+Q_{0}=S T \text { for } S:=x^{2}+y^{2}-z^{2} \text { and } T:=x^{2}+y^{2}-z^{2}+y .
$$

Since $S(D)$ is the wave operator, $S(D)$ admits a continuous linear right inverse on $\mathcal{D}^{\prime}\left(\mathbb{R}^{3}\right)$. By Proposition 3.9, the same holds for $T(D)$, and thus also for the product $\left(P+Q_{0}\right)(D)$.

Remark 4.7. It has been known for some time that the conclusion of Proposition 3.9 does not hold in general if there are singular points of $V\left(P_{m}\right)$ in $S^{n-1}$. The simplest example is provided by the polynomial $P_{2} \in \mathbb{R}[x, y, z]$,

$$
P_{2}(x, y, z)=x y \text {. }
$$

For $Q(x, y, z):=i z$, the operator $\left(P_{2}+Q\right)(D)$ does not admit a continuous linear right inverse in $\mathcal{D}_{(\omega)}^{\prime}\left(\mathbb{R}^{3}\right)$ if the weight function $\omega$ satisfies $\omega(t)=$ $o\left(t^{1 / 2}\right)$. This was shown in Meise, Taylor, and Vogt [15], Example 4.9, but can also be derived from Lemma 3.11.

In [6] we derive new necessary conditions for a given polynomial $P \in$ $\mathbb{C}\left[z_{1}, \ldots, z_{n}\right]$ to satisfy $\mathrm{PL}\left(\mathbb{R}^{n}, \omega\right)$ for a given weight function and we show that these conditions are characterizing when $n=3$. To achieve this, a more refined analysis of the behavior of $V(P)$ in conoids is necessary and $\omega$-hyperbolicity conditions in these conoids play a crucial role.

\section{References}

[1] R.W. Braun, Hörmander's Phragmén-Lindelöf principle and irreducible singularities of codimension 1, Boll. Un. Mat. Ital. (7), 6(3) (1992), 339-348, MR 94b:35012, Zbl 0777.35020.

[2] R.W. Braun, R. Meise and B. A. Taylor, Ultradifferentiable functions and Fourier analysis, Res. Math., 17 (1990), 207-237, MR 91h:46072, Zbl 0735.46022.

[3] _ Algebraic varieties on which the classical Phragmén-Lindelöf estimates hold for plurisubharmonic function, Math. Z., 232 (1999), 103-135, MR 2001d:32048, Zbl 0933.32047.

[4] Characterization of the homogeneous polynomials $P$ for which $(P+Q)(D)$ admits a continuous linear right inverse for all lower order perturbations $Q$, Pacific J. Math., 192 (2000), 201-218, MR 2001d:35030.

[5] _ The geometry of analytic varieties satisfying the local Phragmén-Lindelöf condition and a geometric characterization of the partial differential operators that are surjective on $\mathcal{A}\left(\mathbb{R}^{4}\right)$, to appear in Trans. Amer. Math. Soc.

[6] Characterization of the linear partial differential equations admitting solution operators on Gevrey classes. Preprint.

[7] L. Hörmander, The Analysis of Linear Partial Differential Operators, Vol. II, Grundlehren Math. Wiss., 257, Springer, Berlin, 1983, MR 85g:35002b, Zbl 0521.35002. 
[8] , On the existence of real analytic solutions of partial differential equations with constant coefficients, Invent. Math., 21 (1973), 151-183, MR 49 \#817, Zbl 0282.35015.

[9] B.Ja. Levin, Distribution of Zeros of Entire Functions, Transl. Math. Monograph, 5, Providence, 1980, MR 81k:30011, Zbl 0152.06703.

[10] R. Meise and B. A. Taylor, Phragmén-Lindelöf conditions for graph varieties, Result. Math., 36 (1999), 121-148, MR 2000j:32057, Zbl 0941.32032.

[11] R. Meise, B. A. Taylor and D. Vogt, Characterization of the linear partial differential operators with constant coefficients that admit a continuous linear right inverse, Ann. Inst. Fourier (Grenoble), 40 (1990), 619-655, MR 92e:46083, Zbl 0703.46025.

[12] _ Extremal plurisubharmonic functions of linear growth on algebraic varieties, Math. Z., 219 (1995), 515-537, MR 96j:32019, Zbl 0835.32008.

[13] _ Continuous linear right inverses for partial differential operators on nonquasianalytic classes and on ultradistributions, Math. Nachr., 180 (1996), 213-242, MR 97j:46036, Zbl 0858.46030.

[14] _ Continuous linear right inverses for partial differential operators of order 2 and fundamental solutions in half spaces, Manuscr. Math., 90 (1996), 449-464, MR 97h:35026, Zbl 0876.35023.

[15] _ Phragmén-Lindelöf principles on algebraic varieties, J. Amer. Math. Soc., 11 (1998), 1-39, MR 98j:32007, Zbl 0896.32008.

[16] R. Nevanlinna, Eindeutige Analytische Funktionen, Grundlehren Math. Wiss., 46, Springer, Berlin-New York, 1974, MR 49 \#9165, Zbl 0278.3000.

[17] V.P. Palamodov, A criterion for splitness of differential complexes with constant coefficients, in 'Geometrical and Algebraical Aspects in Several Complex Variables', C.A. Berenstein and D.C. Struppa (Eds.), EditEL, Rende, 1991, 265-291, MR 94d:58137.

Received August 7, 2002. The research of the third author was supported in part by the National Science Foundation under grant number DMS 0070725.

Mathematisches Institut

HEINRICH-HEINE-UNIVERSITÄT

UNIVERSITÄTSSTRASSE 1

40225 DÜSSELDORF

Germany

E-mail address: Ruediger.Braun@uni-duesseldorf.de

Mathematisches Institut

HEINRICH-HEINE-UNIVERSITÄT

UNIVERSITÄTSSTRASSE 1

40225 DÜSSELDORF

Germany

E-mail address: meise@cs.uni-duesseldorf.de

Department of Mathematics

UNIVERSITY OF Michigan

ANn Arbor, MI 48109

E-mail address: taylor@umich.edu 